\title{
Evolutionary Genetics
}

\author{
Ruben C. Arslan \& Lars Penke
}

Institute of Psychology

Georg August University Göttingen

Forthcoming in D. M. Buss (Ed.), Handbook of Evolutionary Psychology

(2nd ed.). New York: Wiley.

September 17, 2014

Corresponding author:

Ruben C. Arslan

Georg August University Göttingen

Biological Personality Psychology and Psychological Assessment

Georg Elias Müller Institute of Psychology

Goßlerstr. 14

37073 Göttingen, Germany

Tel.: +495513920704

Email: ruben.arslan@gmail.com 


\section{Introduction}

When Charles Darwin developed the theory of evolution, he knew nothing about genetics. Hence, one of its biggest weaknesses was that Darwin had to base it on crude ideas of inheritance. Around the same time, Gregor Mendel discovered the laws of inheritance, but the scientific community initially failed to appreciate his work's importance. It was only in the 1930's that Dobzhansky, Fisher, Haldane, Wright, Mayr and others unified genetics and the theory of evolution in the 'modern synthesis'. Still, the modern synthesis was built on a basic understanding of genetics, with genes merely being particulate inherited information. The basics of molecular genetics, like the structure of DNA, were not discovered until the 1950's. When modern evolutionary psychology emerged from ethology and sociobiology in the late 1980 's, it had a strong emphasis on human universals, borne from both the assumption that complex adaptations are monomorphic (or sexually dimorphic) and have to go back to at least the last common ancestor of all humans, and the methodological proximity to experimental cognitive psychology, which tends to treat individual differences as statistical noise. As a consequence, genetic differences between people were marginalized in evolutionary psychology (Tooby \& Cosmides, 1990). Evolutionary psychology and behavior genetics developed nearly orthogonally for over a decade.

Behavior geneticists discovered that virtually every psychological or behavioral difference shows genetic variation (Turkheimer, 2000) and that the molecular genetic underpinnings of most heritable traits are far more complex than assumed in the modern synthesis. Meanwhile, evolutionary 
psychologists increasingly realized the importance of genetic variation, e.g. in models of sexual selection for attractiveness, intelligence and other assumed honest signals of genetic quality (Gangestad \& Simpson, 2000) or heritable variation in life history traits (see Miller \& Penke, 2007). During the last decade, evolutionary genetics gradually gained acceptance among evolutionary psychologists (Buss \& Hawley, 2011; Gangestad \& Yeo, 1997; Penke \& Buss, 2014; Penke, Denissen, \& Miller, 2007), though most still defer fully incorporating the genetic perspective (Miller, 2011).

Evolutionary genetics is concerned with the mechanisms that explain the existence and maintenance of genetic variation in traits. All else equal, one would expect selection to deplete genetic variation in heritable traits related to fitness eventually (Penke et al., 2007). However, such genetic variation is ubiquitous and underlies stable individual differences that play prominent roles in psychological theories, be it as traits under intersexual (e.g. attractiveness, agreeableness, intelligence; (Buss, 1989) and intrasexual selection (masculinity, aggressiveness; Puts, this volume), life history traits, formidability in recalibration theory (Sell, Tooby, \& Cosmides, 2009), sociometer sensitivity (Denissen \& Penke, 2008), perceived vulnerability to infection in the behavioral immune system (Schaller \& Park, 2011), attachment security (Rholes \& Simpson, 2006), or the tendency to show strong reciprocity in cooperation (Fehr, Fischbacher, \& Gächter, 2002). Though these theories ascribe adaptive roles to individual differences, more or less explicitly linking them to fitness, their genetic variation is often taken for granted. 
Evolutionary genetics can help evolutionary psychologists unearth clues to the ultimate reasons behind e.g. humans' cognitive faculties that go beyond what can gleaned through paleontology and archaeology (Enard, Messer, \& Petrov, 2014). This information can have very practical implications, e.g. helping to understand how natural and sexual selection, when altered through changing mores or policy, will affect certain traits.

One aim of this chapter is thus to introduce some of the tools available to researchers in evolutionary genetics. Prior to that, we provide an overview of the forces of evolution and how their interactions can maintain genetic variation. To illustrate the various ways in which evolution can maintain individual differences, we will often invoke specific traits that seem to serve as good, didactically useful examples. The general approach, however, would be applicable to all sorts of traits, including those with relevance to evolutionary psychological theories. Rarely have all possible explanations been weighed explicitly in the literature; we thus tried to refrain from definite statements. With this caveat in mind, we believe that our examples will help evolutionary psychologists make use of the rich theoretical framework that evolutionary genetics provides.

\subsection{Genetic architecture}

Some research in molecular genetics has been carried out with the aim of characterizing the genetic architecture of traits, sometimes also called the genotype-phenotype map (Mackay, 2001). The genetic architecture of a trait can provide important clues to the evolutionary history and the mechanisms 
that govern the maintenance of genetic variation in the trait (Penke et al., 2007). Characterizing the genetic architecture of a quantitative trait would ideally involve its robustness to mutations (canalization) as well as its evolvability. It would also imply gauging its degree of pleiotropy (whether the genes involved also have simultaneous other effects) and the importance of non-additive genetic variation (i.e. epistasis and dominance, variation that does not breed true to the next generation). Unfortunately, many examinations of the genetic architecture are limited to estimates of the number and effect size of involved genetic variants. Often the goal in such examinations is predicting which molecular genetic studies will succeed in the gene hunt and lead to biological pathways and drug targets, not to discover the ultimate, evolutionary explanations for heritable variation in a trait. In this chapter we hope to suggest conceptual approaches to the latter goal.

It may feel like a step back from identifying causative genetic variants, but we feel it is prudent to set aside the exciting prospects of what a successful gene hunt might entail (Chabris et al., 2013) and the different ideas about how we might succeed at that (Graur et al., 2013; Mitchell, 2012), focusing instead on finding common theoretical ground.

Researchers disagree how, if ever, we might explain a substantial portion of the "missing heritability" (Mitchell, 2012), the observable genetic variation left unexplained by molecularly identified genetic variants. The limits of currently available tools can sometimes act as blinders; so that some theoretically plausible genetic architectures are hidden in our blind spots. Fortunately, as rapid technological and statistical development in molecular 
genetics adds to our toolkit, fewer blind spots should impede us. Humility is still very appropriate though, considering fairly principal problems such as the sheer parameter explosion that is encountered when relating genomic sequences to traits (but see Ma, Clark, \& Keinan, 2013).

Neither should we be too eager to jump to the conclusion that our purported core traits will be reflected at the genetic level. For example, Mitchell (2012) argued against the continuous liability-threshold model of psychiatric disease, saying that there truly are discrete disorders, we just tend to group them broadly and arbitrarily. Similar arguments can be construed for the structure of psychological traits like personality and intelligence.

In addition, there are often unresolved questions about the genetic architecture implied by the available evidence. For example, researchers used to believe that selection would reduce genetic variation in fitness traits, driving associated variants to fixation. This seemed to be borne out by low heritability coefficients. However, when researchers realized that fitness traits present a large target for mutation (Merilä \& Sheldon, 1999), they re-examined the same heritability data expressed as the mean-standardized coefficient of variation (an absolute measure) and obtained large estimates of genetic variation. Heritability expressed as a proportion of total variation (a relative measure) had only appeared small in comparison, dwarfed by the large environmental variation (Miller \& Penke, 2007). The conceptualization of fitness traits effectively reversed through a more appropriate statistic for variation. 
Our understanding of how the forces of evolution shape traits' genetic architectures will continue to evolve. Thus, we begin with mechanisms potentially maintaining genetic variation before we discuss methods to identify causative genetic variants.

\section{Forces of evolution}

We will begin by introducing four basic forces that affect genetic variation in populations.

\subsection{Mutation}

All existing genetic variants once arose by mutation. Relative to the 6.4 billion base pairs of the human genomic sequence, mutations are rare events. Beneficial mutations are the rarest of all, the majority likely being neutral to fitness, with deleterious mutations making up the rest. Because the idea of a neutral mutation can be reduced to chance (or drift) being more important for its fate than selection, calling a mutation neutral also depends on its commonness, not just its effect size. A mutation with a small beneficial effect will have its fate determined mostly by chance while it is rare, because chance events can eliminate all copies. Once its frequency rises and in larger populations drift becomes relatively less important, so the mutation will be governed more by selection (Lanfear, Kokko, \& Eyre-Walker, 2014).

The most common mutational event in humans is the change of a single base pair (the letters of the DNA), but there are also deletions, duplications and insertions of base pairs or even longer parts of DNA (copy number 
variants). Aneuploidies (chromosomal aberrations), such as the duplication of chromosome 21 which causes Down syndrome, are rare but massive, accounting for most altered base pairs per birth. Except for aneuploidies, which are well-known to exponentially increase in frequency with advancing maternal age, all types of mutations occur more often on the paternal side, and increasingly so with advancing paternal age at conception (Campbell \& Eichler, 2013). Proximately, this is often attributed to the continuous division of cells in the paternal but not maternal germline (Kong et al., 2012), but ultimate explanations such as Bateman's principle (male investment in each offspring is lower) should be kept in mind (Stearns, 2005).

\subsection{Selection}

Selection occurs when there is heritable variation in fitness. Natural selection is frequently broken down into different subcategories. One grouping distinguishes positive, directional selection (favoring increases), disruptive selection, (favoring extremes), and stabilizing selection, (favoring decreased variation in a trait). Another grouping considers survival and sexual selection separately. Sometimes this is differentiated further into "episodes of selection". Survival selection could e.g. be divided into the chances of an ovum to be released in ovulation, sperm fertilizing an ovum, a zygote implanting, the pregnancy being carried to term (Stearns, 2005), and surviving birth, to reproductive age, and further. Sexual selection might be divided into the odds of finding and attracting a mate, outcompeting same-sex rivals, the number of mates, the number of offspring per mate, and the fitness and number of offspring in the next few generations. Often the mistaken 
impression that selection has diminished in humans is, on closer inspection, limited to factors affecting perinatal and postnatal survival selection, with little heed paid to components of sexual selection.

\subsubsection{Correlated selection, genetic hitchhiking and pleiotropy}

Genetic variants are not independently selected for. As the term "genetic hitchhiking" vividly implies, alleles can hitch a ride on the coattails, or haplotype, of a neighboring allele that is being selected for or against. The chances of inheriting a specific gene from a parent are not independent from those of its neighbors because we inherit genes in chunks. Over generations, recombination breaks haplotypes apart. Long unbroken haplotypes signal strong recent selection for a new mutation, because the neighboring alleles of a beneficial mutation are "swept" along on the coattails before recombination can break them apart (known as a "hard sweep"). Shorter unbroken haplotypes can signal selection on standing (pre-existing) genetic variation ("soft sweeps"; Pritchard, Pickrell, \& Coop, 2010). Two or more alleles that usually co-occur (are in "linkage disequilibrium") and thus form a haplotype can have different, even opposing effects on fitness. Until recombination breaks them apart, they cannot be selected for independently.

Alleles experience correlated selection not only through proximity. Even a variant at a single locus can have multiple, pleiotropic effects on fitness via different phenotypic consequences. It can also make sense to distinguish fitness effects of an allele in different episodes of selection. For example, a mutation may be selected for pre-meiotically in the testes, but lead to Apert syndrome later on (Choi, Yoon, Calabrese, \& Arnheim, 2008). 


\subsection{Genetic drift}

Luck plays a lead role when numbers are small. If there are few carriers of even a highly beneficial genetic variant, random events can eliminate all of them. Similarly, a deleterious variant can be fixated by chance, or a beneficial rare variant can randomly get lost in recombination. Either way, a gene variant may drift to fixation or extinction just by chance. If all variants at a locus are common (because no single variant is infrequent and the population is large), the law of large numbers implies that it will take long before either drifts to fixation. In humans, a comparatively extremely low genetic diversity points to genetic bottlenecks having been an important instance of drift (Gazave, Chang, Clark, \& Keinan, 2013). Bottlenecks may occur through migration, such as when founder populations emigrated to North America, or when population sizes decreased dramatically through harsh conditions such as droughts, epidemics or ice ages. If the resulting population is small and not diverse (e.g. a clan), even beneficial alleles from the parent population may be lost through drift.

\subsection{Gene flow (or migration)}

When individuals carrying certain alleles move from one group to another, the frequency of alleles in each group also changes. This process is distinguished from unsystematic genetic drift, because relevant genetic variants may differentially influence the propensity to migrate and the success in each group and environment. 


\section{Maintenance mechanisms}

Prolonged directional or stabilizing selection on a trait will deplete its genetic variance. The mechanisms that maintain heritable variation in a trait can be understood as equilibria or trade-offs between the forces of evolution that change allele frequencies: selection, mutation, genetic drift, and gene flow. In some cases it may seem as if evolution should lead to alternative genetic architectures with fewer trade-offs. Note that evolution is not over and that optimal solutions may not always be sufficiently better to be selected over merely adequate ones, which is e.g. why we still have blind spots in our eyes.

\subsection{First-order mechanisms}

\subsubsection{Mutation-selection balance (MSB)}

Mutations continuously emerge. If they are entirely neutral, they are invisible to selection and may drift or hitchhike to extinction or fixation. But if they are deleterious, purifying selection will act against them. We rarely hear of dominant lethal mutations because they tend to be eliminated within one generation. Huntington's disease, which develops after the age of reproduction, is one example to the contrary.

If a trait is genetically complex, as most traits of interests to evolutionary psychologists likely are, many genes will be involved, not all of which play a crucial role. Hence, some deleterious mutations will be selected against less intensely and might linger for a few generations. If the mutational target size of a trait (the number of associated genetic loci) is large, mutations affecting the trait will accumulate, so that individuals carry a certain mutational load. 
Thus, variation in a trait such as physical attractiveness can be maintained even though it is likely under directional selection. In research on the genetics of autism spectrum disorders, new mutations appear to explain about $15 \%$ of cases (Devlin \& Scherer, 2012), though this should not be equated with the part that MSB plays for autism, which may well be larger owing to older, inherited mutation load. Debate revolves around the number of genes likely to be involved in a trait and on the question whether rare, recent or common, older mutations mostly disrupt such genes (Gazave et al., 2013).

\subsubsection{Mutations in balance with stabilizing vs. directional selection}

Traits under mutation-selection balance can be meaningfully differentiated further. If increases in a trait are linked to increased fitness (directional selection), new mutations should usually cause a decline in the trait. This assumption is implicit in most studies of MSB.

If fitness is instead linked to a certain optimum in a trait, it is said to be under stabilizing selection. Stabilizing selection acts to increase robustness to deleterious mutations, for example by increasing genetic redundancy. For sexually recombining species, such as ours, it has also been suggested that increased mutational robustness need not imply a decrease in the evolvability of a trait (its potential to react to selection): redundancy reduces the selection pressure on individual variants and thus allows variation to build up in the back-up copy, creating a playground for genetic innovation. In this case, new mutations should cause comparatively smaller deviations from the optimum and might lead us to miss genetic associations if we focus on directional declines. The optimum would be expected to be the mean of a trait, at least in 
traits that were not subject to recent environmental changes. The shape of the eye might be an example of this exception: myopia (short-sightedness, elongated eyes) is more common than hyperopia (early-onset farsightedness, shortened eyes), but the preponderance of myopia sufferers might be attributed to changes in our environment, in which near work became common and time outdoors decreased (Mingroni, 2004). To determine the not immediately visible optima of psychological traits, researchers could draw on associations of trait levels with survival and mate preferences as proxies of fitness consequences.

\subsubsection{Balancing selection}

We now introduce a class of balancing mechanisms. In all of them, one selection pressure is counteracted by another in a different location, time, developmental stage, social environment or intraindividual genetic context.

\subsubsection{By spatial environmental heterogeneity (migration-selection balance):}

Humans can experience different selection pressures in different environments. Selection by location need not be limited to selection pressures such as varying solar intensity (Norton et al., 2007) or altitude (Simonson et al., 2010), though these examples are best-characterized.

Because personality may affect one's penchant for travel, migration can support spatial balancing selection: If those who want to see the world keep leaving their home island for the mainland, the remaining islanders may end up less open to experience on average (Ciani \& Capiluppi, 2011). Selection would also reduce variance in openness if sedentary islanders did not 
occasionally interbreed with visitors from the mainland. This sort of recurring gene flow can maintain variation in openness. Similarly, sociability supports migration tendencies from rural to urban areas (Jokela, Elovainio, Kivimäki, \& Keltikangas-Järvinen, 2008). In scenarios such as these genetic variation is maintained because people within a population select themselves into the environments for which they are best adapted. Such niche picking (also known as active gene-environment correlation) is potentially a strong force in the maintenance of genetic variation in humans (Penke, 2010). In the population as a whole, no trait or underlying genetic variant would effectively be favored, thus the selection pressures would balance.

Because cultural and other environmental explanations are hard to disentangle from genetically based psychological differences between populations, we advocate a cautious approach to this controversial topic. Some jump to premature conclusions about major genetic differences and even superiority based on flimsy evidence such as fairly high within-group heritability coefficients, but a balanced view of the evidence shows how difficult explaining group differences genetically is (Berg \& Coop, 2014).

Because of humans' ecological dominance and concomitant capacity to shape the environment to their needs (niche construction), Penke, Denissen, and Miller (2007; Penke, 2010) argued that the most important fluctuating aspect that humans need to adapt to is their social environment.

\subsubsection{By social environment (negative frequency-dependent selection)}

There are three morphs (types) of male common side-blotched lizards (Uta 
stansburiana), and three alleles at one Mendelian locus govern their throat color and concomitant behavior. Blue-throated males guard one mate and territory. Their mates can be stolen by larger, aggressive, orange-throated males, who keep large territories and multiple mates. Because they do not guard their mates well, they are vulnerable to having their mates stolen by yellow-throated males, who pretend to be female to sneakily gain access. This non-transitive mating game has been compared to rock-paper-scissors (Sinervo \& Lively, 1996) and leads to oscillations in which the least common morph becomes more common in the next generation.

Biological sex is probably the most familiar morph under such negative frequency-dependent selection (NFDS) in humans, as the rarer sex becomes more desirable and thus has reproductive advantages due to mating market forces (Del Giudice, 2012). NFDS has also been invoked to explain primary psychopathy (Mealey, 1995), personality traits (Penke et al., 2007) and, perhaps most fruitfully, immunity to parasites (Sutton, Nakagawa, Robertson, \& Jamieson, 2011).

If psychopathy were under frequency-dependent selection, we might, through altered policy, lower the equilibrium frequency of psychopaths within few generations (Mealey, 1995).

\subsubsection{Over time (generations)}

If selection fluctuates over time more quickly than is needed for trait alleles to be driven to either fixation or extinction, variation can be maintained in oscillations. For example, if sex ratios in populations naturally fluctuate over 
time, genetic variation in personality traits that lead to better mating outcomes in one sex can be maintained by balancing selection (Del Giudice, 2012). If the fluctuations are predictable, selection should act to create genetically fixed conditional (facultative) strategies instead, a rich topic for life history theory (Nettle, Frankenhuis, \& Rickard, 2013; Penke, 2009, 2010).

\subsubsection{Over time (ontogenetic development)}

Earlier we mentioned an allele that proliferates in the testes, but leads to disease (Choi et al., 2008). Negatively correlated selection across developmental stages is also plausible for quantitative traits. For instance, large heads may support cognitive ability in later life, but they complicate birth (Miller \& Penke, 2007). Selection should favor traits that are not subject to such trade-offs, but especially in conjunction with fluctuations of the fitness effects at different developmental stages, variation could be maintained.

\subsubsection{By genetic variant at other loci (epistasis)}

An allele may have a beneficial or deleterious effect only in the presence or absence of other genetic variants. The sheer complexity of considering all the interactions in conjunction with the already large number of variants in the human genome has led some to propose that evolution would lead to mainly additive and even modularized variation in certain traits (W.G. Hill, Goddard, \& Visscher, 2008), but epistasis might also be missed owing to insufficient statistical power. 


\subsubsection{By genetic variant at the same locus (overdominance, heterozygote advantage, selection-drift balance)}

Consider a polymorphism, such as the one involved in sickle-cell anemia. Two copies of the polymorphism make blood cells sickle-shaped under lowoxygen conditions and typically lead to premature death. But having only one copy (heterozygosity) confers greater resistance to malaria. Individuals from areas in which malaria was a strong selective pressure are more often carriers of the sickle-cell polymorphism. Heterozygotes have a selective advantage over homozygotes with either allele and so the sickle-cell allele can persist in the population at equilibrium frequency.

These equilibria are not stable: an allele that has the benefits but not the disadvantages will easily displace its competitor. We expect to see overdominance especially under strong, recent selection, such as that incurred by epidemics.

\subsubsection{Mutation-drift balance (Selective/ancestral neutrality)}

If mutations affecting a neutral trait arise so frequently that some linger before they drift out of existence, we expect genetic variation in this trait to linger as well. Because of the nature of genetic drift, existing, entirely neutral polymorphisms would linger longer in large populations. Because most human DNA is nonfunctional junk, which is not conserved through purifying selection, most mutations are neutral (Graur et al., 2013). One's first intuition might then be that most human individual differences are selectively neutral or "evolutionary noise" (Tooby \& Cosmides, 1990). However, a commonly variable trait that is phenotypically visible to selection is less likely to be 
entirely neutral. This is especially the case since we tend to be interested in traits because they have predictive value for consequential life outcomes such as reproductive success, and thus evolutionary fitness. Additionally, because populations are larger nowadays, selection is more efficient, and will usually be stronger than drift (Penke et al., 2007).

In humans, with their rapidly changing culture and environment and with their rapidly increasing population size (Gazave et al., 2013), we might want to pay special heed to traits that used to be selectively neutral or nearly so, but no longer are. These are traits where we might expect natural selection to rapidly deplete genetic variation. Because traits under mutation-drift balance have a repository of standing variation and because selection is stronger than drift, it can decrease previously maintained variation.

A potential candidate for an ancestrally neutral psychological trait may be our preference for rising early or late: Our circadian rhythm is entrained to a universal source of light, the sun, in areas with little artificial light. With more artificial light, individuals' circadian rhythms become more variable (Wright et al., 2013), and such differences are moderately heritable (Barclay, Eley, Buysse, Archer, \& Gregory, 2010). Possibly what we see here is cryptic genetic variation, revealed only under artificial light. Without it the lack of variation in light exposure within populations might have meant that heritable differences were not visible, even though psychological differences that would have influenced self-exposure to artificial light already existed. 


\subsection{Mechanisms implicating more than one trait a time}

In this section we will consider mechanisms that lead to the impression that there is heritable variability in a trait, but which are best understood in conjunction with other mechanisms and traits.

\subsubsection{Mechanisms related to pleiotropy and hitchhiking}

When genes are pleiotropic (affect multiple traits) or in linkage (in close proximity to each other on a chromosome), genetic correlations among traits can appear. There are ways to discover genetic correlations and to analyze contemporary selection on multiple correlated traits (Stearns, Byars, Govindaraju, \& Ewbank, 2010), but few studies have tried to do so for human evolutionary history.

The best-characterized examples of antagonistic pleiotropy arise in conjunction with biological sex. Traits like facial masculinity may be more adaptive in one sex than the other, but the respective alleles spend half their careers in each sex (A.J. Lee et al., 2014). Another important class of pleiotropic interactions may arise through the body's limited energy budget, especially that available for immune, brain and gut functions. As a consequence, selection cannot optimize either trait, eventually resulting in a continuum of equally fit trait combinations maintained in the population.

\subsubsection{Reactive heritability}

Not every trait with heritable individual differences needs to be subject to some sort of balancing mechanism itself. Instead, it could be calibrated to another heritable trait (Tooby \& Cosmides, 1990). For example, Lukaszewski 
and Roney (2011) posited that extraversion might be calibrated to one's physical attractiveness and strength. Hence, we would find the signature of mutation-selection balance when studying extraversion in isolation, but would come to different conclusions when examining developmental and situational calibration of extraverted behaviour to one's relative strength and attractiveness.

If they are not fixed at birth, we should not presume the primacy of physical traits. For example, we know that myopia appears to be linked to the amount of time children spend outdoors (Sherwin et al., 2012), but the substantial heritability estimates for myopia have led some researchers to downplay environmental explanations for the recent increase in myopia incidence (Mingroni, 2004). But if myopia heritability is partly reactive to children's heritable proclivity for outdoor play and if some children spend less time outdoors in recent times, which is plausible, these findings could be reconciled.

\section{The Evolutionary Genetics Toolkit}

In this section, we will introduce the growing toolkit that is available to evolutionary geneticists. These tools were assembled from both quantitative and molecular genetics, as well as evolutionary psychology. We will note what these tools can be used for, and how they are sometimes misused, but acknowledge how all of these methods make their contributions. 


\subsubsection{Twin and family studies}

Twin studies are one of the oldest tools available and have withstood the test of time (Conley, Rauscher, Dawes, Magnusson, \& Siegal, 2013). They rely on the key difference between monozygotic (identical) twins and dizygotic (fraternal) twins: identical twins share all of their genes, while fraternal twins share on average half of the genes that were variable between their parents. A central result from twin studies is usually a heritability estimate, though the rich data from twin and family studies can answer many other questions too. The concept hails from plant and animal breeding, where it is used to predict response to artificial selection.

Estimates of heritability derived from twin studies have held up remarkably well when re-examined using different family relationships (e.g. parents, siblings, half- and adopted siblings) and can be easily extended to novel data such as the sometimes numerous offspring of sperm donors. In cases where selection is fairly clear-cut, estimates of heritability have borne out their usefulness as predictors of the response to selection. For example, children of sperm donors are taller in a manner consistent with their mothers' selection on donor height (J.C. Lee, 2013).

Usually things are not so tidy: Heritability estimates from twin studies often include some non-additive variation, i.e. variation that will not "breed true" to the next generation. Moreover, environmental confounds can make it hard to isolate an effect of selection, as the initiators of the Scottish Mental Survey discovered in 1947 when they attempted to show a decline of intelligence through differential fertility and found an increase instead (Ramsden, 2007). 
Humans simply do not behave like crops on a field or cattle in a breeding facility; they actively choose mates and both choose and modify their environments. This decreases the value of heritability estimates as more than a proof that genetic differences play a role in observable phenotypic variation (Johnson, Penke, \& Spinath, 2011).

High heritability in twin studies has often been misunderstood to imply that a trait cannot be changed. To the contrary, species-typical universals such as two-leggedness have virtually zero heritability, because the underlying genes rarely vary. On the other hand, some gene-environment interactions were not apparent before the relevant environment changed: For example, developing phenylketonuria, a disease causing intellectual disability, depends on consuming phenylalanine, which was a universal part of our diet before its damaging effects in some individuals became known.

\subsubsection{Linkage studies}

Linkage studies, which identify larger genetic segments that segregate according to disease status in a pedigree, have been useful tools in the identification of "simple" Mendelian disorders, where single genes have major effects. They might also help once we learn to tell apart phenotypically similar diseases that we now group as complex psychiatric disorders (Mitchell, 2012). Linkage studies for most psychological variation have been characterized as a let-down. Still, they ruled out a suggested genetic architecture: If there were, for example, a single genetic locus causing human psychopathy (i.e. an exploitative social strategy) in analogy with the aforementioned sneaky sideblotched lizard, linkage patterns would have led to its identification. 


\subsubsection{Candidate gene studies}

Candidate gene studies look for the association of a specific genetic locus with the trait of interest. By hypothesizing which locus may be involved $a$ priori, they avoid correcting for multiple comparisons and can thus use smaller samples than the similar, but exploratory genome-wide association paradigm. They have come under intense criticism, because of non-replications and general doubts whether there is sufficient theory to predict candidate genes (loannidis, Trikalinos, Ntzani, \& Contopoulos-loannidis, 2003).

Some recent studies, however, successfully employ candidate gene approaches, implicating candidate gene sets and apparently building on stronger theory than before. For example, W. D. Hill and colleagues (2014) reported and replicated an association of intelligence with variation in genes involved in one of the postsynaptic density complexes that have been implicated in cognitive functioning. Through pre-registration of candidate genes, researchers could easily end disagreements and distrust whether their studies deserve the label of confirmatory research and concomitant relaxation of false discovery rates. Unfortunately, this is seldom done.

\subsubsection{Genome-wide association studies (GWAS)}

GWAS assess the status of individuals on around a million genetic loci across the genome that are commonly variable in the population. While GWAS directly assess only around $0.033 \%$ of the human genome this way, linkage disequilibrium makes the assessed variants fairly exhaustive markers of common genetic variation, which is then related to the variation in the trait of interest. GWAS require large samples and have been early adopters of 
harsh significance thresholds to account for the number of multiple comparisons (loannidis et al., 2003).

GWAS have been successful in the identification of some of the genes that matter for pigmentation, some medical disorders, height, and recently schizophrenia (Schizophrenia Working Group of the Psychiatric Genomics Consortium, 2014). Yet, for most psychological traits, especially normal variation, they rarely identified replicable associations (Chabris et al., 2012, 2013). This is often framed negatively, but GWAS effectively ruled out genetic architectures involving few common variants of medium-to-large effects for all psychological traits studied this way so far. Some researchers have advocated ever larger samples in order to potentially identify huge sets of genetic variants with individually miniscule effect sizes, while others argue that theory predicts only effects of questionable practical relevance and that family-based designs are better-suited (Mitchell, 2012).

\subsubsection{Using sequenced exomes and genomes in association studies}

Sequencing refers to identifying every single base pair in someone's genome, not just a few commonly polymorphic loci, as in GWAS. When sequencing is limited to protein-coding genes (ca. 1-2\% of the whole genome), this subset is called the exome. The exome constitutes a more manageable amount of data, and has been considered promising for clinical variation. However, much of it is conserved between species and a lot of recent selection has operated on promoters outside the exome (Enard et al., 2014; The 1000 Genomes Project Consortium, 2012), making exome variation 
a less likely candidate for contributing to the genetic architecture of psychological traits in the normal range (Marioni et al., 2014).

With the amounts of data generated by genome sequencing, entirely exploratory research would not be useful due to the sample sizes required to filter chance findings. Integrating prior knowledge, such as annotations on regions with a signature of recent selection or expression in the brain (Ma et al., 2013), or alternatively relying on summary indices of rare genetic variants, a direct operationalization of mutation load (Marioni et al., 2014), may make such data manageable.

\subsubsection{Genomic prediction and genome-wide complex trait analysis (GCTA)}

A method formerly used primarily to predict breeding value in domestic animals has recently become popular in human genetics under the name GCTA (Yang et al., 2011). The general method estimates distant relatedness (less than fourth cousins) between individuals in the general population on the basis of common genetic variants, as provided by GWAS. Unlike GWAS, this method does not identify individual important loci. Instead the distant relatedness is used to infer a heritability score akin to that known from twin studies, but based solely on molecular data. After many GWAS failed to identify loci associated with psychological traits, GCTA provided a means of showing that the genotype data was actually informative: it can validate heritability estimates and be used to enable marker-assisted breeding (though this application is unlikely in humans), even if it does not identify causative genes and hence provides no foothold to find biological pathways. A 
frequently raised objection is that GCTA heritability estimates might be spurious, driven by the resemblance of distantly genetically related individuals for non-genetic reasons, such as similar environments because of shared ancestry and migration history. Researchers working with GCTA acknowledge such confounds, the discussion revolves mostly about whether the corrections are sufficient (Conley et al., 2014; Yang et al., 2011).

Some researchers also doubt whether finding high GCTA heritability implies that the infinitesimal model of many common variants of tiny effect applies, especially when debilitating disorders are under study (Mitchell, 2012). Maybe more agreement can be fostered by a shift to delineating a fully-featured genetic architecture, acknowledging the balanced forces enumerated in this chapter.

\subsection{Paternal age effects}

By sequencing and comparing the genomes of both parents and an offspring, Kong and colleagues (2012) convincingly demonstrated that the number of newly occurred single nucleotide variants in offspring can almost entirely be accounted for by the father's age at conception. Thus, paternal age can be used as a proxy variable to infer the effect of new mutations. To isolate this effect, the fact that human reproductive timing is not governed by chance has to be statistically controlled. Initially reported negative associations between paternal age and intelligence in the normal range (Malaspina et al., 2005) have not been replicated in later studies. Controlling parental intelligence, an important predictor of reproductive timing, may account for 
some of the observed heterogeneity of effects (Arslan, Penke, Johnson, lacono, \& McGue, 2014). Employing sibling comparison designs also led to the disappearance of paternal age effects on intelligence, while a strong association with attention deficit hyperactivity disorder became visible only with sibling controls (D’Onofrio et al., 2014).

Properly isolated, paternal age effects can provide evidence for a trait being under mutation-selection balance. In addition, they can be useful to predict the effect of increasingly delayed reproduction in the industrialized world (Sartorius \& Nieschlag, 2010), net of potentially more modifiable aspects such as parental investment.

\subsection{Genome and exome triplets and quads}

When the entire exomes or even genomes of parent-offspring trios are sequenced, it becomes possible to count new mutations, i.e. alleles that neither parent carried. By assessing which haplotype a mutation lies on, it is also possible to identify the parent of origin. Then, mutation counts can predict e.g. intellectual disability (Rauch et al., 2012) and recurring mutations can be used to zero in on causative genes.

Exome quads (both parents and two offspring) have been used in autism genetics. Using genome annotations, lossifov and colleagues (2012) estimated which mutations interrupted genes. By also sequencing unaffected siblings whose genomes were recombined from a common parental pool, they could isolate the effect of having more disrupted genes. Studies on autism genetics tried to isolate the effect of new mutations from assortative mating by 
only considering families without a familial history of autism and through sibling comparisons. These molecular genetic studies corroborate earlier results of autism increasing with paternal age.

\subsection{Inbreeding depression and outbreeding elevation}

Inbreeding depression refers to a fitness decrease in offspring of consanguinous unions. Consanguinous parents (second cousins and closer) and their offspring make up about $10 \%$ of the world's population, though their prevalence has been predicted to decline (Bittles \& Black, 2009). Franssen (2009) reported a linear negative relationship between offspring mental ability and consanguinity ranging from second-cousin-marriages to incest. Such associations are confounded by many unobserved common causes. For example, lower parental education can, via lower mobility, increase the likelihood of marrying relatives and thus inflate estimates of inbreeding depression. The family history and cultural prevalence of consanguinity (e.g. in clans and castes) affect inbreeding coefficients too, so that estimates based on just two generations can be off (Bittles, 2010).

Outbreeding elevation, also known as hybrid vigor or heterosis, refers to the increased phenotypic quality of the offspring of genetically more distant parents. This phenomenon is very familiar to plant and animal breeders. Mules may be the most iconic hybrids and hybrid maize the most frequently consumed. The vigor does not necessarily translate to evolutionary fitness: mules are valued beasts of burden, but frequently infertile. This is because too distant genetic relationships between parents can break up co-adapted gene 
complexes during recombination, hence breaking vital functions such as the ability to reproduce. A bit of both may have happened when modern humans and Neanderthals interbred (Sankararaman et al., 2014). Hybrid vigor can also occur when inbreeding ends: Mixed-breed dogs have higher life expectancy than most purebreds (O'Neill, Church, McGreevy, Thomson, \& Brodbelt, 2013). Mingroni (2004) proposed that urbanization and generally less sedentism led to decreased inbreeding and might be partial causes for the recent increases in height and intelligence in industrialized countries.

\subsection{Runs of homozygosity}

Analogously to GCTA, which employs DNA-based subtle relatedness to validate twin studies' estimate of heritability, runs of homozygosity $(\mathrm{ROH})$ are an attempt to characterize subtle inbreeding on a molecular level.

If long stretches of a diploid genome are homozygous, i.e. both strands of DNA have the same variants, we can infer that closely related individuals have bred. If many shorter stretches are homozygous, we can infer ancient relatedness (Kirin et al., 2010). The genomic approach has the benefit that inbreeding over several generations can be characterized, though it is important to supplement this with knowledge of the history of endogamous marriage, founder effects and population bottlenecks (Bittles, 2010). Homozygosity appears to play a role not only in well-characterized recessive disorders such as cystic fibrosis, but also for traits like personality (Verweij et al., 2012, 2014). Power and colleagues (2013) found a zero-to-slightlypositive association between $\mathrm{ROH}$ burden and intelligence, which conflicts 
with (possibly more biased) pedigree-based estimates of inbreeding effects (Franssen, 2009).

\subsection{Relations with fitness (lifetime reproductive success) and mate preferences}

It may seem as if we have so far neglected the obviously relevant effects of traits on fitness measures in this chapter. This is because, with some exceptions (e.g. pervasive developmental disorders), it is difficult to establish that the same association has persisted over evolutionary time and is thus indicative of the balancing mechanism which primarily upheld variation in a trait. We lack historical data for psychological traits, and many associations between normal variation and fitness estimated nowadays could be fickle. Contemporary selection on human individual differences is interesting in itself (Stearns et al., 2010), but we expect evolutionary genetics, among other disciplines, to answer the question "Why did humans evolve to be this way?"

In the age of widespread effective contraception it can be argued that mate preferences and choices are better-preserved indicators of sexual selection than correlations with reproductive success. In addition to being more immediately assessable than lifetime reproductive success, mate preferences have been shown to be relatively culturally invariant (Buss, 1989), unlike total fertility. Perinatal and postnatal survival selection plausibly have decreased in intensity since the advent of hygiene, modern health care, less frequent infanticide and lower infant and maternal mortality. Still, a large number of pregnancies are not carried to term and many debilitating, previously lethal 
genetic conditions, such as severe disability, may now be sexually selected against owing to lower attractiveness in the mating market.

\subsection{Correlations with indicators of developmental stability}

Bilateral fluctuating asymmetry (FA) of the body is presumed to be an indicator of developmental stability, operating under the assumption that mutation-free organisms in good condition will be more symmetrical (Polak, 2003). Correlations with FA are thus assumed to provide an indirect way to tap a trait's association with mutation load. This paradigm is prevalent in evolutionary psychology and somewhat plagued by publication bias (Van Dongen \& Gangestad, 2011). Hardly any studies take a molecular or population genetic approach to fluctuating asymmetry in humans. Future studies should more directly examine an association of developmental stability indicators with rare genetic variant burden, paternal age, or consanguinity before correlations with FA can be deemed valid proxies for tapping 'good genes'. Pre-registration of studies could foster greater trust, especially that of scientists in adjacent domains such as genetics.

\section{Conclusion and outlook}

Evolution by natural selection occurs as long as there is heritable variation related to differential fitness in the population. The evidence for both is ubiquitous even today, posing the question why so much genetic variation persisted. Genetic variance is influenced by mutation, selection, drift and migration, and combinations of these four forces can yield balanced states in 
which it is maintained. This has been known since the modern synthesis in the 1930's, but our understanding of the molecular genetics underlying these processes has radically progressed. We are increasingly able to learn about the genetic architecture underlying psychological traits. Although the resulting picture will not be as simple as most researchers assumed even a few years ago, it can eventually provide insights about the evolutionary history and the selection pressures currently acting on these traits (Penke et al., 2007).

The evolutionary genetic toolkit includes complementary tools from molecular, behavior genetics and classical evolutionary psychology. Every available method has so many caveats that only converging evidence can enable us to single out theories as tenable. Unfortunately, even closely neighboring disciplines do not often lend each other tools and insights. For example, pure life history models of psychopathology (Del Giudice, Klimczuk, Traficonte, \& Maestripieri, 2014) are inconsistent with the accumulating evidence that mutation load plays a major role in the autism and schizophrenia spectra (Andreassen et al., 2014). Research on runs of homozygosity and mutation load could verify assumptions inherent in studies on fluctuating asymmetry. We need to subject our favored evolutionary explanations to tools from outside our own respective fields. Different disciplines can find it hard to properly evaluate and trust results outside their own field, especially if there is publication bias. Data and discussion brought to bear on the matter may have ideological baggage and bias (Ramsden, 2007), as researchers on e.g. intelligence or inbreeding, where science is easily conflated with moral judgments, know well. However, we can restore 
trust in areas plagued by bias (e.g. candidate gene and fluctuating asymmetry studies) through pre-registration, replication, collaboration in consortia, and greater transparency. Such quality badges can be recognized even if the exact details are beyond us (Miller, 2011). By embracing such superior scientific standards we can protect our theories from the charge of being "justso stories".

It is encouraging, however, that all these approaches share a common evolutionary meta-theory, which could help to integrate knowledge acquired using diverse tools and build a common understanding. We have referenced numerous positive examples throughout this chapter. Mutual assistance and understanding should lead not only to agreement on the existence of heritable individual differences, but on the mechanisms maintaining them. Even where we identify genetic architectures that make it hard for us to detect important causative genes (e.g. an infinitesimal number of causative genes of small effect, genetic heterogeneity, or epistasis), there is a lot to be gleaned from understanding maintaining mechanisms. These mechanisms are not idle theory; they have practical applications. Policy and mores already exert influence on demography, reproductive timing and selective pressures. We do not need to know specific genetic variants to predict what will happen to autism incidence if people reproduce later, nor to characterize the role of assortative mating and consanguinity in the age of online dating, nor to understand the impact of anciently constant selective forces suddenly swayed by new technology. 
Where we identify traits with a genetic architecture conducive to identifying causative genes, many doors open for vertical integration (Y.W. Lee, Gould, \& Stinchcombe, 2014) with biology and neuroscience: we can study pathways, develop drugs and genetic screenings, examine molecular signatures of selection and demographic history (Enard et al., 2013), use Mendelian randomization techniques (Smith \& Ebrahim, 2004) to identify modifiable causes of disease, and make inferences about earlier hominids' psychological characteristics on the basis of shared polymorphisms.

Darwin knew nothing about the genetics underlying evolution, but our ever more detailed understanding allows us to fully embrace the potential of merging evolutionary theory with genetics. Evolutionary genetics enriches evolutionary psychology by providing a theoretical framework and tools to integrate individual differences and recent evolution (Penke, 2010), and thus ultimately an understanding of why we are the way we are and how we became that way.

\section{References}

Arslan, R.C., Penke, L., Johnson, W., lacono, W.G., \& McGue, M. (2014). The effect of paternal age on offspring intelligence and personality when controlling for paternal trait level. PLOS ONE, 9, e90097.

Barclay, N.L., Eley, T.C., Buysse, D.J., Archer, S.N., \& Gregory, A.M. (2010). Diurnal preference and sleep quality: Same genes? A study of young adult twins. Chronobiology International, 27, 278-296. 
Berg, J.J., \& Coop, G. (2014). A population genetic signal of polygenic adaptation. PLoS Genetics, 10, e1004412.

Bittles, A.H. (2010). Consanguinity, genetic drift, and genetic diseases in populations with reduced numbers of founders. In M. R. Speicher, A. G. Motulsky, \& S. E. Antonarakis (Eds.), Vogel and Motulsky's Human Genetics (pp. 507-528). Springer Berlin Heidelberg.

Bittles, A.H., \& Black, M.L. (2009). Consanguinity, human evolution, and complex diseases. Proceedings of the National Academy of Sciences, $107,1779-1786$.

Buss, D.M. (1989). Sex differences in human mate preferences: Evolutionary hypotheses tested in 37 cultures. Behavioral and Brain Sciences, 12, $1-49$.

Buss, D.M., \& Hawley, P.H. (2011). The evolution of personality and individual differences. Oxford University Press.

Campbell, C.D., \& Eichler, E.E. (2013). Properties and rates of germline mutations in humans. Trends in Genetics, 1-10.

Chabris, C.F., Hebert, B.M., Benjamin, D.J., Beauchamp, J., Cesarini, D., van der Loos, M., ... Laibson, D. (2012). Most reported genetic associations with general intelligence are probably false positives. Psychological Science, 23, 1314-1323.

Chabris, C.F., Lee, J.J., Benjamin, D.J., Beauchamp, J.P., Glaeser, E.L., Borst, G., ... Laibson, D.I. (2013). Why it is hard to find genes associated with social science traits: Theoretical and empirical considerations. American Journal of Public Health, 103, S152-S166. 
Choi, S.-K., Yoon, S.-R., Calabrese, P., \& Arnheim, N. (2008). A germ-lineselective advantage rather than an increased mutation rate can explain some unexpectedly common human disease mutations. Proceedings of the National Academy of Sciences of the United States of America, 105, 10143-8.

Ciani, A.C., \& Capiluppi, C. (2011). Gene flow by selective emigration as a possible cause for personality differences between small islands and mainland populations. European Journal of Personality, 25, 53-64.

Conley, D., Rauscher, E., Dawes, C., Magnusson, P.K.E., \& Siegal, M.L. (2013). Heritability and the equal environments assumption: Evidence from multiple samples of misclassified twins. Behavior Genetics, 43, $415-426$.

Conley, D., Siegal, M.L., Domingue, B.W., Mullan Harris, K., McQueen, M.B., \& Boardman, J.D. (2014). Testing the key assumption of heritability estimates based on genome-wide genetic relatedness. Journal of Human Genetics, 59, 342-345.

D’Onofrio, B.M., Rickert, M.E., Frans, E., Kuja-Halkola, R., Almqvist, C., Sjölander, A., ... Lichtenstein, P. (2014). Paternal age at childbearing and offspring psychiatric and academic morbidity. JAMA Psychiatry. doi:10.1001/jamapsychiatry.2013.4525

Del Giudice, M. (2012). Sex ratio dynamics and fluctuating selection on personality. Journal of Theoretical Biology, 297, 48-60.

Del Giudice, M., Klimczuk, A.C.E., Traficonte, D.M., \& Maestripieri, D. (2014). Autistic-like and schizotypal traits in a life history perspective: 
diametrical associations with impulsivity, sensation seeking, and sociosexual behavior. Evolution and Human Behavior, 35, 415-424.

Denissen, J.J.A., \& Penke, L. (2008). Motivational individual reaction norms underlying the Five-Factor model of personality: First steps towards a theory-based conceptual framework. Journal of Research in Personality, 42, 1285-1302.

Devlin, B., \& Scherer, S.W. (2012). Genetic architecture in autism spectrum disorder. Current Opinion in Genetics \& Development, 22, 229-237.

Enard, D., Messer, P.W., \& Petrov, D.A. (2014). Genome-wide signals of positive selection in human evolution. Genome Research, 24, 885895.

Fehr, E., Fischbacher, U., \& Gächter, S. (2002). Strong reciprocity, human cooperation, and the enforcement of social norms. Human Nature, 13, $1-25$.

Franssen, D. (2009). Is there a linear relationship between inbreeding and mental ability?: A meta-analysis. Retrieved from http://dare.uva.nl/document/152307

Gangestad, S.W., \& Simpson, J.A. (2000). The evolution of human mating: Trade-offs and strategic pluralism. Behavioral and Brain Sciences, 23, 573-587.

Gangestad, S.W., \& Yeo, R.A. (1997). Behavioral genetic variation, adaptation and maladaptation: an evolutionary perspective. Trends in Cognitive Sciences, 1, 103-108. 
Gazave, E., Chang, D., Clark, A.G., \& Keinan, A. (2013). Population growth inflates the per-individual number of deleterious mutations and reduces their mean effect. Genetics, 195, 969-978.

Graur, D., Zheng, Y., Price, N., Azevedo, R.B.R., Zufall, R.A., \& Elhaik, E. (2013). On the immortality of television sets: "Function" in the human genome according to the evolution-free gospel of ENCODE. Genome Biology and Evolution, 5, 578-590.

Hill, W.D., Davies, G., van de Lagemaat, L.N., Christoforou, A., Marioni, R.E., Fernandes, C.P.D., ... Deary, I.J. (2014). Human cognitive ability is influenced by genetic variation in components of postsynaptic signalling complexes assembled by NMDA receptors and MAGUK proteins. Translational Psychiatry, 4, e341.

Hill, W.G., Goddard, M.E., \& Visscher, P.M. (2008). Data and theory point to mainly additive genetic variance for complex traits. PLoS Genetics, 4, e1000008.

Ioannidis, J.P., Trikalinos, T.A., Ntzani, E.E., \& Contopoulos-loannidis, D.G. (2003). Genetic associations in large versus small studies: an empirical assessment. The Lancet, 361, 567-571.

Iossifov, I., Ronemus, M., Levy, D., Wang, Z., Hakker, I., Rosenbaum, J., ... Wigler, M. (2012). De novo gene disruptions in children on the autistic spectrum. Neuron, 74, 285-299.

Johnson, W., Penke, L., \& Spinath, F.M. (2011). Heritability in the era of molecular genetics: Some thoughts for understanding genetic 
influences on behavioural traits. European Journal of Personality, 25, 254-266.

Jokela, M., Elovainio, M., Kivimäki, M., \& Keltikangas-Järvinen, L. (2008). Temperament and migration patterns in Finland. Psychological Science, 19, 831-837.

Kirin, M., McQuillan, R., Franklin, C.S., Campbell, H., McKeigue, P.M., \& Wilson, J.F. (2010). Genomic runs of homozygosity record population history and consanguinity. PLOS ONE, 5, e13996.

Kong, A., Frigge, M.L., Masson, G., Besenbacher, S., Sulem, P., Magnusson, G., ... Stefansson, K. (2012). Rate of de novo mutations and the importance of father's age to disease risk. Nature, $488,471-475$.

Lanfear, R., Kokko, H., \& Eyre-Walker, A. (2014). Population size and the rate of evolution. Trends in Ecology \& Evolution, 29, 33-41.

Lee, A.J., Mitchem, D.G., Wright, M.J., Martin, N.G., Keller, M.C., \& Zietsch, B.P. (2014). Genetic factors that increase male facial masculinity decrease facial attractiveness of female relatives. Psychological Science, 25, 476-484.

Lee, J.C. (2013). Quantitative genetics in the postmodern family of the donor sibling registry. Retrieved from https://www.donorsiblingregistry.com/sites/default/files/files/dissertation (1).pdf

Lee, Y.W., Gould, B.A., \& Stinchcombe, J.R. (2014). Identifying the genes underlying quantitative traits: A rationale for the QTN programme. $A O B$ Plants, plu004. 
Lukaszewski, A.W., \& Roney, J.R. (2011). The origins of extraversion: joint effects of facultative calibration and genetic polymorphism. Personality \& Social Psychology Bulletin, 37, 409-421.

Ma, L., Clark, A.G., \& Keinan, A. (2013). Gene-based testing of interactions in association studies of quantitative traits. PLoS Genet, 9, e1003321.

Mackay, T.F.C. (2001). The genetic architecture of quantitative traits. Annual Review of Genetics, 35, 303-339.

Malaspina, D., Reichenberg, A., Weiser, M., Fennig, S., Davidson, M., Harlap, S., ... Knobler, H.Y. (2005). Paternal age and intelligence: implications for age-related genomic changes in male germ cells. Psychiatric Genetics, 15, 117-125.

Marioni, R.E., Penke, L., Davies, G., Huffman, J.E., Hayward, C., \& Deary, I.J. (2014). The total burden of rare, non-synonymous exome genetic variants is not associated with childhood or late-life cognitive ability. Proceedings of the Royal Society B: Biological Sciences, 281, 20140117.

Mealey, L. (1995). The sociobiology of sociopathy: An integrated evolutionary model. Behavioral and Brain Sciences, 18, 523-541.

Merilä, J., \& Sheldon, B.C. (1999). Genetic architecture of fitness and nonfitness traits: empirical patterns and development of ideas. Heredity, 83, 103-109.

Miller, G.F. (2011). Are pleiotropic mutations and Holocene selective sweeps the only evolutionary-genetic processes left for explaining heritable variation in human psychological traits? In D. M. Buss \& P. H. Hawley 
(Eds.), The evolution of personality and individual differences (p. 376). New York: Oxford University Press.

Miller, G.F., \& Penke, L. (2007). The evolution of human intelligence and the coefficient of additive genetic variance in human brain size. Intelligence, 35, 97-114.

Mingroni, M.A. (2004). The secular rise in IQ: Giving heterosis a closer look. Intelligence, 32, $65-83$.

Mitchell, K.J. (2012). What is complex about complex disorders? Genome Biology, 13, 237.

Nettle, D., Frankenhuis, W.E., \& Rickard, I.J. (2013). The evolution of predictive adaptive responses in human life history. Proceedings of the Royal Society B: Biological Sciences, 280.

Norton, H.L., Kittles, R.A., Parra, E., McKeigue, P., Mao, X., Cheng, K., ... Shriver, M.D. (2007). Genetic evidence for the convergent evolution of light skin in Europeans and East Asians. Molecular Biology and Evolution, 24, 710-722.

O’Neill, D.G., Church, D.B., McGreevy, P.D., Thomson, P.C., \& Brodbelt, D.C. (2013). Longevity and mortality of owned dogs in England. The Veterinary Journal, 198, 638-643.

Penke, L. (2009). Adaptive developmental plasticity might not contribute much to the adaptiveness of reproductive strategies. Behavioral and Brain Sciences, 32, 38-39.

Penke, L. (2010). Bridging the gap between modern evolutionary psychology and the study of individual differences. In D. M. Buss \& P. H. Hawley 
(Eds.), The evolution of personality and individual differences (pp. 243279). New York: Oxford University Press.

Penke, L., Denissen, J.J.A., \& Miller, G.F. (2007). The evolutionary genetics of personality. European Journal of Personality, 21, 549-587.

Polak, M. (2003). Developmental instability: causes and consequences. Oxford University Press.

Power, R.A., Nagoshi, C., DeFries, J.C., Donnelly, P., Barroso, I., Blackwell, J.M., ... Plomin, R. (2013). Genome-wide estimates of inbreeding in unrelated individuals and their association with cognitive ability. European Journal of Human Genetics. doi:10.1038/ejhg.2013.155

Pritchard, J.K., Pickrell, J.K., \& Coop, G. (2010). The genetics of human adaptation: Hard sweeps, soft sweeps, and polygenic adaptation. Current Biology, 20, R208-R215.

Ramsden, E. (2007). A differential paradox: The controversy surrounding the Scottish mental surveys of intelligence and family size. Journal of the History of the Behavioral Sciences, 43, 109-134.

Rauch, A., Wieczorek, D., Graf, E., Wieland, T., Endele, S., Schwarzmayr, T., ... Strom, T.M. (2012). Range of genetic mutations associated with severe non-syndromic sporadic intellectual disability: an exome sequencing study. Lancet, 380, 1674-1682.

Rholes, W.S., \& Simpson, J.A. (2006). Adult attachment: Theory, research, and clinical implications. Guilford Press. 
Sankararaman, S., Mallick, S., Dannemann, M., Prüfer, K., Kelso, J., Pääbo, S., ... Reich, D. (2014). The genomic landscape of Neanderthal ancestry in present-day humans. Nature, 507, 354-357.

Sartorius, G.A., \& Nieschlag, E. (2010). Paternal age and reproduction. Human Reproduction Update, 16, 65-79.

Schaller, M., \& Park, J.H. (2011). The behavioral immune system (and why it matters). Current Directions in Psychological Science, 20, 99-103.

Schizophrenia Working Group of the Psychiatric Genomics Consortium. (2014). Biological insights from 108 schizophrenia-associated genetic loci. Nature, 511, 421-427.

Sell, A., Tooby, J., \& Cosmides, L. (2009). Formidability and the logic of human anger. Proceedings of the National Academy of Sciences, 106, $15073-15078$.

Sherwin, J.C., Reacher, M.H., Keogh, R.H., Khawaja, A.P., Mackey, D.A., \& Foster, P.J. (2012). The association between time spent outdoors and myopia in children and adolescents: A systematic review and metaanalysis. Ophthalmology, 119, 2141-2151.

Simonson, T.S., Yang, Y., Huff, C.D., Yun, H., Qin, G., Witherspoon, D.J., ... Ge, R. (2010). Genetic evidence for high-altitude adaptation in Tibet. Science, 329, 72-75.

Sinervo, B., \& Lively, C.M. (1996). The rock-paper-scissors game and the evolution of alternative male strategies. Nature, 380, 240-243. 
Smith, G.D., \& Ebrahim, S. (2004). Mendelian randomization: prospects, potentials, and limitations. International Journal of Epidemiology, 33, $30-42$.

Stearns, S.C. (2005). Issues in evolutionary medicine. American Journal of Human Biology, 17, 131-140.

Stearns, S.C., Byars, S.G., Govindaraju, D.R., \& Ewbank, D. (2010). Measuring selection in contemporary human populations. Nature Reviews Genetics, 11, 611-622.

Sutton, J.T., Nakagawa, S., Robertson, B.C., \& Jamieson, I.G. (2011).

Disentangling the roles of natural selection and genetic drift in shaping variation at MHC immunity genes. Molecular Ecology, 20, 4408-4420.

The 1000 Genomes Project Consortium. (2012). An integrated map of genetic variation from 1,092 human genomes. Nature, 491, 56-65.

Tooby, J., \& Cosmides, L. (1990). On the universality of human nature and the uniqueness of the individual: The role of genetics and adaptation. Journal of Personality, 58, 17-67.

Turkheimer, E. (2000). Three laws of behavior genetics and what they mean. Current Directions in Psychological Science, 9, 160-164.

Van Dongen, S., \& Gangestad, S.W. (2011). Human fluctuating asymmetry in relation to health and quality: a meta-analysis. Evolution and Human Behavior, 32, 380-398.

Verweij, K.J.H., Abdellaoui, A., Veijola, J., Sebert, S., Koiranen, M., Keller, M.C., ... Zietsch, B.P. (2014). The association of genotype-based 
inbreeding coefficient with a range of physical and psychological human traits. PLOS ONE, 9, e103102.

Verweij, K.J.H., Yang, J., Lahti, J., Veijola, J., Hintsanen, M., Pulkki-Råback, L., ... Zietsch, B.P. (2012). Maintenance of genetic variation in human personality: testing evolutionary models by estimating heritability due to common causal variants and investigating the effect of distant inbreeding. Evolution, 66, 3238-3251.

Wright, K.P., McHill, A.W., Birks, B.R., Griffin, B.R., Rusterholz, T., \& Chinoy, E.D. (2013). Entrainment of the human circadian clock to the natural light-dark cycle. Current Biology, 23, 1554-1558.

Yang, J., Manolio, T.A., Pasquale, L.R., Boerwinkle, E., Caporaso, N., Cunningham, J.M., ... Hayes, M.G. (2011). Genome partitioning of genetic variation for complex traits using common SNPs. Nature Genetics, 43, 519-525. 